\title{
Cooling with a Squeeze
}

\section{A newly designed alloy exhibits a "colossal" elastocaloric effect-a temperature change under strain-making it a good candidate for an environmentally friendly type of cooling.}

\section{by Jaka Tušek* and Nini Pryds ${ }^{\dagger}$}

$\mathrm{T}$ oday's society thrives on cooling. In Europe, for example, $70 \%$ of the food is chilled or frozen, while in the US, air conditioners in people's homes consume more electricity than all of Africa. The demand for cooling is only expected to rise [1], along with its exacerbating effects on global warming, creating a push to find alternatives to the century-old and relatively inefficient vapor compression cooling technology in use today [2]. A promising option is to employ elastocaloric solids. These materials change their temperature in response to a mechanical stress, a property that can be used to pull heat from an object in thermal contact. Daoyong Cong of the University of Science and Technology Beijing and colleagues now report that they have designed and synthesized an alloy whose temperature rises and falls more than $30 \mathrm{~K}$ when strained and then released-the largest reversible elastocaloric effect observed so far.

The vapor compression cooling that chills our food and buildings involves circulating a compressed refrigerant, which is forced to suddenly expand so it can carry away heat. The "exergy" efficiency of this method-the ratio between the actual and theoretical efficiency-is relatively low (about 20\%.) Moreover, refrigerants in current vapor compressors can be hazardous or cause pollution, and the compression process generates noise and vibrations. Cooling technology based on so-called caloric effects has a potentially higher efficiency and no environmental hazards. Caloric materials undergo a heat-releasing (exothermic) phase transition when exposed to an external field that is magnetic, electric, or based on mechanical strain. The reverse process-a heat-absorbing (endothermic) reaction-occurs when the field is removed. These combined effects can be used in a cooling cycle if the field drives the transition faster than the material can release heat to or absorb heat from its surroundings (Fig. 1). This cycle involves four steps: applying the field so that the material's tem-

\footnotetext{
* Faculty of Mechanical Engineering, University of Ljubljana, Ljubljana, Slovenia

$\dagger$ Department of Energy Conversion and Storage, Technical University of Denmark, Roskilde, Denmark
}

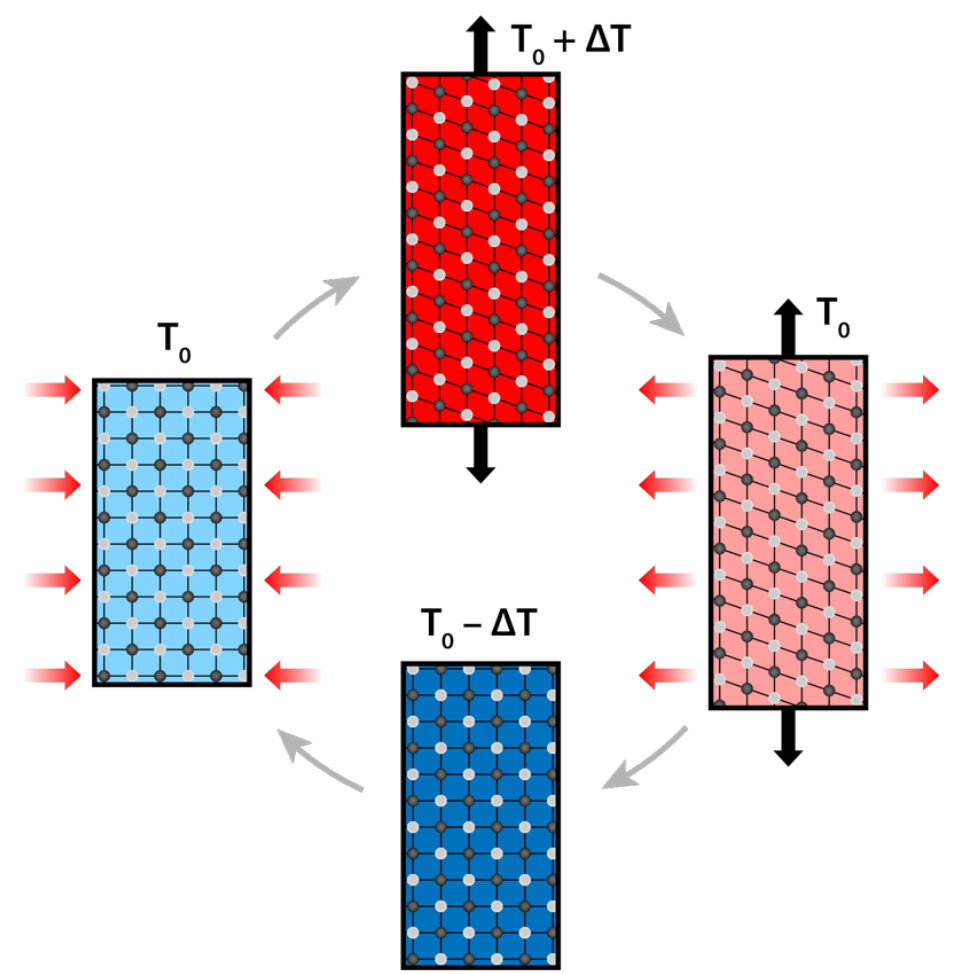

Figure 1: Clockwise from top: the four steps in a refrigeration cycle based on an elastocaloric material. Mechanical strain causes a structural phase transition, heating the material. The material is then allowed to release heat to its surroundings and to drop to a lower temperature. When the strain is released, the crystal structure transitions and the material drops in temperature, allowing it to pull in heat and cool its surroundings. (APS/Alan Stonebraker)

perature will rise; allowing the material's heat to flow out; removing the field so that the material cools down below its original temperature; and allowing the material to cool its surroundings. For application in a cooling device, the elastocaloric temperature change should ideally be as high as possible and reversible, so that the cooling cycle can be repeated many times.

So far, researchers have devoted most of their attention to the magnetocaloric effect-a change in temperature induced by a magnetic field. But 20 years of studying magnetocaloric 
materials has failed to lead to a commercial cooling product that could satisfy global needs [3]. Magnetocaloric cooling is also expensive because the materials and the magnets needed to control them mostly rely on rare-earth elements. By comparison, elastocaloric materials, which change temperature under strain, are cheaper to make and control, and they exhibit larger temperature changes. The strain drives what's known as a martensitic transition, where the material's atoms suddenly "slide" into a new crystal structure (Fig. 1). The elastocaloric effect has been known for almost 40 years [4], but its potential use for cooling (or for heating) has only been recognized in the past decade because of the development of new and effective materials [5]. The ensuing studies have been so promising that in 2014, the US Department of Energy declared elastocaloric technology to be the best alternative to vapor compression [2].

So far, the largest elastocaloric effects (above $20 \mathrm{~K}$ ) have been demonstrated in shape-memory alloys-metals that, once molded, will snap back to their original shape when heated. Most of those alloys were optimized for applications based on the shape memory effect or the related phenomenon of superelasticity, not elastocalorics. The work by Cong and colleagues brings the elastocaloric property to the fore [6]. The team focused on a class of polycrystalline nickel manganese (NiMn) based alloys, which they shaped into cylinders that were stressed along their long axes. At a few hundred megapascals of stress, the materials exhibit a martensitic phase transition with a corresponding volume change of up to $2 \%$. In this type of material, large volume changes correspond to large temperature changes, so the team adjusted the alloy composition-the ratio of $\mathrm{Ni}$ to $\mathrm{Mn}$ and titanium (Ti) - to find the maximum volume change. And they added in boron (B) to increase the compounds' stability under high stress. Following this search strategy, the team zeroed in on $\left(\mathrm{Ni}_{50} \mathrm{Mn}_{31.5} \mathrm{Ti}_{18.5}\right)_{99.8} \mathrm{~B}_{0.2}$, in which they measured a reversible elastocaloric temperature change of $31.5 \mathrm{~K}-\mathrm{a}$ higher change than that found for any known elastocaloric, magnetocaloric, or electrocaloric solid.

These numbers are impressive. However, if history is any guide, many new materials never escape the "valley of death" between discovery and an actual commercial device. Still, recent years have provided a few proof-of-concept elastocaloric devices. These range from miniature "single-stage" devices, which rely on direct thermal contact between an elastocaloric material and a heat sink or source [7], to more complex devices, where a fluid (like water) acts as a heat transfer medium [8-10]. So far, the most promising cooling (and heating) characteristics have been demonstrated with the fluid-transfer approach in so-called active regenerative elastocaloric devices $[9,10]$. Experiments measured a temperature span of $20 \mathrm{~K}$ and a specific heating power of $800 \mathrm{~W} / \mathrm{kg}$ of elastocaloric material-enough to cool down a small office on a hot summer day. The devices also have an energy efficiency approaching that of vapor compression.
Ultimately, the market potential of elastocaloric technology rests on addressing two big challenges. First, the material should be able to withstand millions of loading cycles, which is especially challenging as the material must have a thin shape (film, wire, or tube) to ensure fast and efficient heat transfer with its surroundings. Second, new driver mechanisms are needed to mechanically load the elastocaloric material. The ideal driver applies the necessary force efficiently, and it can recapture the work released when the elastocaloric material unloads. Efficient work recovery is one of the main conditions for realizing efficient elastocaloric technology, and it hasn't been met yet. Nonetheless, with their design strategy, Cong and colleagues have equipped researchers with better tools to discover colossal elastocaloric effects in other materials, and with any luck, better devices in the future.

This research is published in Physical Review Letters.

\section{REFERENCES}

[1] M. Isaac and D. P. van Vuuren, "Modeling global residential sector energy demand for heating and air conditioning in the context of climate change," Energy Policy 37, 507 (2009).

[2] W. Goetzler, R. Zogg, J. Young, and C. Johnson, "Energy savi ngs potential and RD opportunities for non-vapor-compression HVAC technologies," Navigant Consulting, Inc., prepared for U.S. Department of Energy (2014).

[3] A. Kitanovski, J. Tušek, U. Tomc, U. Plaznik, M. Ožbolt, and A. Poredoš, Magnetocaloric energy conversion: From theory to applications (Springer, New York, 2015).

[4] C. Rodriguez and L. C. Brown, "The thermal effect due to stress-induced martensite formation in -CuAINi single crystals," Metall. Mater. Trans. A 11, 147 (1980).

[5] E. Bonnot, R. Romero, L. Mañosa, E. Vives, and A.Planes, "Elastocaloric effect associated with the martensitic transition in shape-memory alloys," Phys. Rev. Lett. 100, 125901 (2008).

[6] D. Cong et al., "Colossal elastocaloric effect in ferroelastic NiMn-Ti alloys," Phys. Rev. Lett. 122, 255703 (2019).

[7] H. Ossmer, F. Wendler, M. Gueltig, F. Lambrecht, S. Miyazaki, and M. Kohl, "Energy-efficient miniature-scale heat pumping based on shape memory alloys," Smart Mater. Struct. 25, 085037 (2016).

[8] S. Qian, Y. Wu, J. Ling, J. Muehlbauer, Y. Hwang, I. Takeuchi, and R. Radermacher, "Design, development and testing of a compressive thermoelastic cooling system," Proceedings of the 24th IIR International Congress of Refrigeration (ICR 2015) (International Institute of Refrigeration, Paris, 2015)Vol. 1.

[9] J. Tušek, K. Engelbrecht, D. Eriksen, S. Dall'Olio, N. Pryds, "A regenerative elastocaloric heat pump," Nat. Energy 1, 16134 (2016).

[10] K. Engelbrecht, J. Tušek, D. Eriksen, T. Lei, C.-Y. Lee, J. Tušek, and N. Pryds, "A regenerative elastocaloric device: Experimental results," J. Phys. D 50, 424006 (2017).

10.1103/Physics. 12.72 